\title{
A novel approach to create a tunable Fano resonance with an extinction ratio over $40 \mathrm{~dB}$
}

\author{
Ang $\mathrm{Li}^{1,2}$ and Wim Bogaerts ${ }^{1,2}$ \\ ${ }^{1}$ Photonics Research Group, Ghent University-IMEC, Department of Information Technology, Ghent 9052, Belgium. \\ ${ }^{2}$ Center for Nano- and Biophotonics (NB-photonics), Ghent University, Ghent 9052, Belgium. \\ Email: ang.li@ugent.be
}

\begin{abstract}
We experimentally demonstrate a novel method to make a tunable Fano resonance. Based on a silicon microring with two tunable reflectors inside, we are able to generate a tunable Fano resonance with maximum extinction ratio over 40 $\mathrm{dB}$ and a slope rate over $700 \mathrm{~dB} / \mathrm{nm}$.

Index Terms-Fano resonance, Ring resonator, Integrated reflector, Silicon photonics.
\end{abstract}

\section{INTRODUCTION}

Resulting from the interference between a continuum background mode and a discrete resonance mode[1], a Fano resonance can exhibit an extremely sharp slope, which can benefit many applications such as optical switches, optical sensors, laser cavities and electro-optical modulators. Only a tiny wavelength shift is needed to achieve a large power variation, which usually translates into high efficiency, low power consumption and high speed. Silicon photonics, on the other hand, is one of the most promising platforms to implement those applications due to its ultra high index contrast and CMOS compatibility, which allows very compact device footprint and large-scale, low-cost manufacture.

Various approaches have been reported to demonstrate a Fano resonance in silicon photonics, but a satisfying solution towards a fully integrated circuit with an efficient tuning mechanism to generate a fully tunable Fano resonance with adequate extinction ratio (ER) and slope rate is still missing[2], [3]. In this paper we propose a novel method based on an integrated photonics circuit on silicon-on-insulator platform. With simple thermo-optic heaters, we can achieve efficient tuning of the generated Fano resonance with a maximum extinction ratio (ER) over $40 \mathrm{~dB}$ and a corresponding slope rate more than $400 \mathrm{~dB} / \mathrm{nm}$. In terms of the maximum slope rate, a value over $700 \mathrm{~dB} / \mathrm{nm}$ is observed at some resonances with an extinction ratio around $36 \mathrm{~dB}$. The maximum observed ER and slope rate, to our best knowledge, are the largest realized so far in an integrated circuit.

\section{Simulation}

The schematic of our device is given in Fig. 1. It can be described as a silicon ring resonator with two integrated tunable reflectors inside (indicated in red). These two reflectors will form a Fabry-Perot (FP) cavity embedded in the ring resonator. As a consequence, the mode from FP cavity will interfere with the resonance mode of the ring resonator at its drop port. When the FP mode is in slowly varying condition
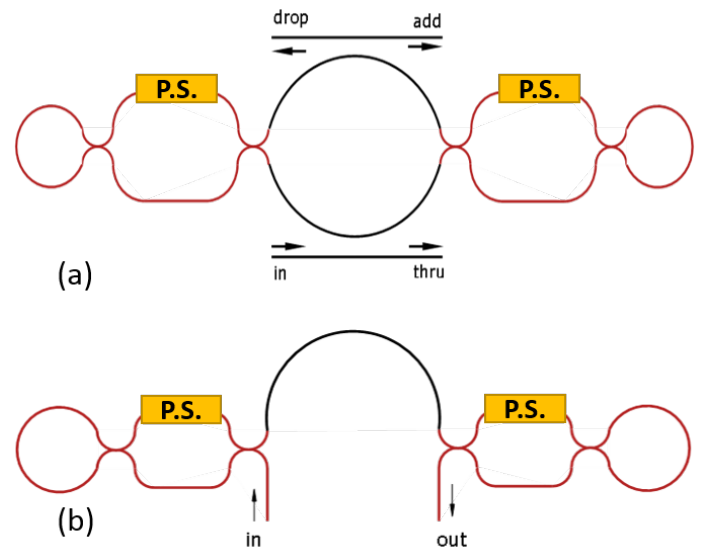

Fig. 1. Schematics of our proposed device (a) and the Fabry-Perot cavity formed by the reflectors (b)

(low $Q$ factor and large free spectral range), the interference of these two modes will generate Fano resonance. This condition can be achieved by controlling the reflectivity of the two reflectors, as the FP mode depends strongly on the reflectivity. As for the reflector, which is a loop-ended Mach-ZenhderInterferometer, its reflectivity can be tuned from 0 to almost $100 \%$ by adding $\frac{\pi}{2}$ phase shift on one arm of the MZI[4].

We simulate our device using the optical circuit simulatorCaphe by Luceda Photonics. In Fig. 2(a) and (c), the spectra of the embedded FP cavity formed by the two tunable reflectors are shown. Clearly, by adding different phase shifts on one arm of the reflector, the FP output can be tuned between very sharp mode and slowly varying mode. Note that for simplicity, we only tune one reflector, while in practice both reflectors can be tuned independently. In Fig. 2(b) and (d) we plot the corresponding output of the complete device. Due to the intentional reflections inside the ring, the output of the device shows strong resonance splitting. The difference in the shift rates of the two peaks within one split resonance is consistent with former observation and has already been well explained[4]. Fano resonances are observed in multiple conditions. Generally speaking, there is a tendency that slowly varying FP mode will lead to a Fano resonance with larger extinction ratio.

The devices were fabricated through Europractice MultiProject-Wafer (MPW) service on 200mm CMOS line of 

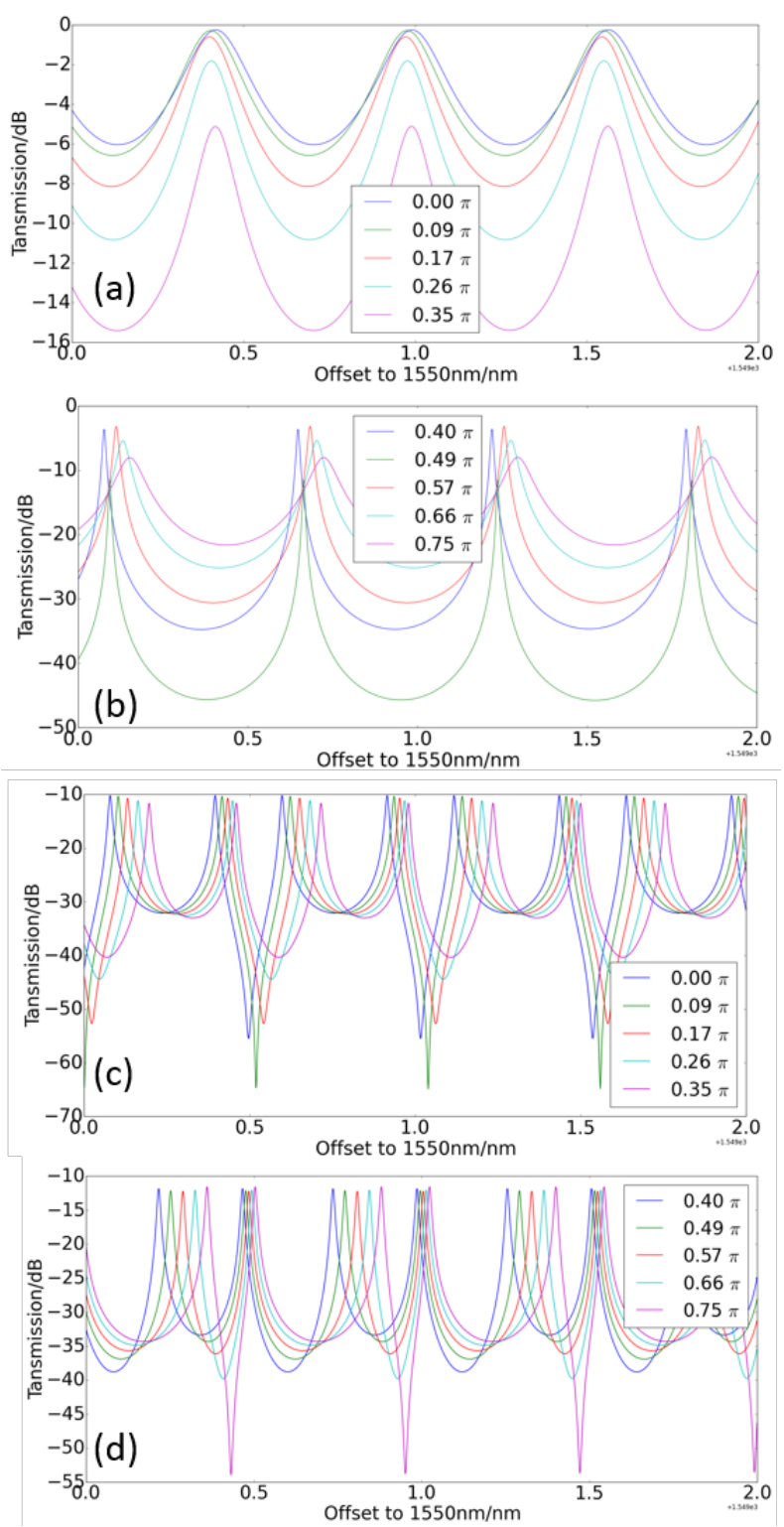

Fig. 2. Figures showing the simulated spectra of the embedded FP cavity (a) and (c), as well as the complete device (b) and (d).

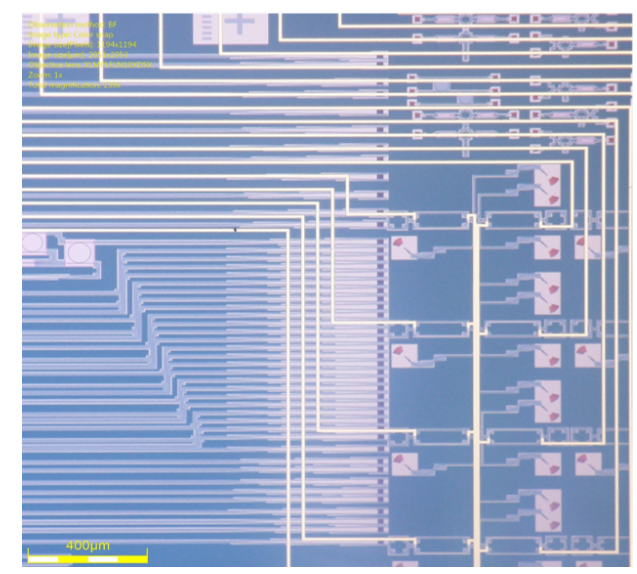

Fig. 3. Microscopic image of the fabricated devices.
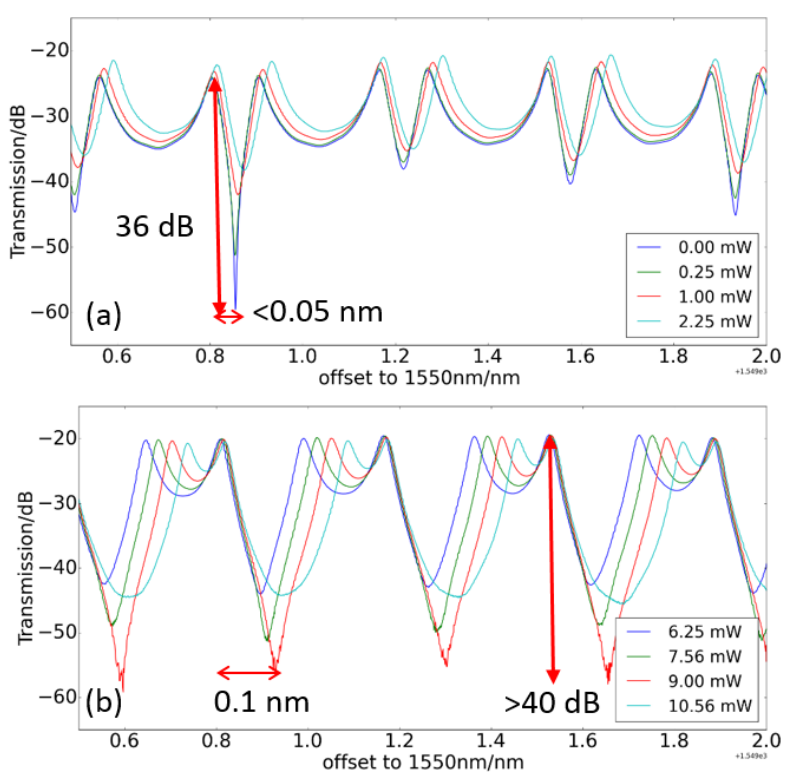

Fig. 4. The measurement results match the simulation results quite well and demonstrate that an extinction ratio over $40 \mathrm{~dB}$ can be achieved with a corresponding slope sharper than $400 \mathrm{~dB} / \mathrm{nm}$. While for the slope rate, it can be more than $700 \mathrm{~dB} / \mathrm{nm}$.

IMEC, shown in Fig. 3. The waveguide is designed to have a cross-section about $450 \mathrm{~nm} \times 220 \mathrm{~nm}$ to ensure single mode operation. Top oxide cladding is used to protect the structures as well as to support the metal heaters processed in our own cleanroom. The heaters consist of a $100 \mathrm{~nm}$ thick Titanium layer which serves as resistive element and a $500 \mathrm{~nm}$ Gold layer as the electrical wires and pads. The resistance of the $200 \mu \mathrm{m}$ long heaters are measured to be around $1 \mathrm{k} \Omega$.

We plot the experimental data in Fig. 4. The Fano resonance can be tuned by feeding power into the heaters. The maximum ER and slope rate observed can be over $40 \mathrm{~dB}$ and $700 \mathrm{~dB} / \mathrm{nm}$, respectively. Less than $7 \mathrm{~mW}$ power is adequate to tune the ER from lowest value ( $15 \mathrm{~dB}$ ) to its maximum ( over $40 \mathrm{~dB}$ ). The tuning efficiency can be further improved by using more efficient phase shifters, for instance, side heaters made of doped silicon waveguides. Besides the tuning of the ER, the location of each Fano resonance can also be tuned by global temperature control or implementing another phase shifter in the rest part of the device, for instance the ring waveguide or the loop waveguide of the reflector.

\section{REFERENCES}

[1] U. Fano, "Effects of configuration interaction on intensities and phase shifts," Physical Review, vol. 124, no. 6, p. 1866, 1961.

[2] W. Zhang, W. Li, and J. Yao, "Optically tunable fano resonance in a grating-based fabry-perot cavity-coupled microring resonator on a silicon chip," Optics letters, vol. 41, no. 11, pp. 2474-2477, 2016.

[3] T. Hu, P. Yu, C. Qiu, H. Qiu, F. Wang, M. Yang, X. Jiang, H. Yu, and J. Yang, "Tunable fano resonances based on two-beam interference in microring resonator," Applied Physics Letters, vol. 102, no. 1, p. 011112, 2013.

[4] A. Li and W. Bogaerts, "Fundamental suppression of backscattering in silicon microrings," Optics Express, vol. 25, no. 3, pp. 2092-2099, 2017. 